\title{
Splenic CD11C(+) cells derived from semi-immune mice protect naïve mice against experimental cerebral malaria
}

\author{
Lam Q Bao ${ }^{1 *}$, Dang M Nhi ${ }^{1}$, Nguyen T Huy ${ }^{2}$, Mihoko Kikuchi ${ }^{3}$, Tetsuo Yanagi ${ }^{4}$, Shinjiro Hamano ${ }^{5}$ \\ and Kenji Hirayama ${ }^{*}$
}

\begin{abstract}
Background: Immunity to malaria requires innate, adaptive immune responses and Plasmodium-specific memory cells. Previously, mice semi-immune to malaria was developed. Three cycles of infection and cure ('three-cure') were required to protect mice against Plasmodium berghei (ANKA strain) infection.

Methods: C57BL/6 J mice underwent three cycles of $P$. berghei infection and drug-cure to become semi-immune. The spleens of infected semi-immune mice were collected for flow cytometry analysis. CD11c(+) cells of semiimmune mice were isolated and transferred into naïve mice which were subsequently challenged and followed up by survival and parasitaemia.

Results: The percentages of splenic CD4(+) and $\operatorname{CD11c(+)}$ cells were increased in semi-immune mice on day 7 post-infection. The proportion and number of B220(+)CD11C(+)low cells (plasmacytoid dendritic cells, DCs) was higher in semi-immune, three-cure mice than in their naïve littermates on day 7 post-infection ( 2.6 vs $1.1 \%$ and 491,031 vs 149,699, respectively). In adoptive transfer experiment, three months after the third cured $P$. berghei infection, splenic CD11C(+) DCs of non-infected, semi-immune, three-cure mice slowed Plasmodium proliferation and decreased the death rate due to neurological pathology in recipient mice. In addition, anti-P. berghei lgG1 level was higher in mice transferred with $\mathrm{CD} 11 \mathrm{c}(+)$ cells of semi-immune, three-cure mice than mice transferred with $\mathrm{CD} 11 \mathrm{c}(+)$ cells of naive counterparts.
\end{abstract}

Conclusion: $\mathrm{CD} 11 \mathrm{c}(+)$ cells of semi-immune mice protect against experimental cerebral malaria three months after the third cured malaria, potentially through protective plasmacytoid DCs and enhanced production of malaria-specific antibody.

Keywords: Semi-immune, CD11C(+) DCs, Plasmacytoid DCs, Cerebral malaria, Malaria-specific antibody

\section{Background}

Immunity to malaria in humans is a step-wise process that spans invasion of sporozoites into the body to development of erythrocytic parasites. This process involves humoral and cellular responses of innate immune cells (e.g., macrophages, dendritic cells (DCs)) and adaptive IFN- $\gamma$-producing CD4(+) and $\mathrm{CD} 8(+) \mathrm{T}$ cells [1]. Malaria infection at the individual and community levels is affected by acquired immunity. A person exposed to repeated Plasmodium infections may

\footnotetext{
* Correspondence: lamquocbao@nagasaki-u.ac.jp; hiraken@nagasaki-u.ac.jp 'Department of Immunogenetics, Institute of Tropical Medicine, Nagasaki University, Nagasaki, Japan

Full list of author information is available at the end of the article
}

develop a partially protective immunity. Such 'semi-immune' persons often can be infected by malaria but rarely manifest the typical severe symptoms [2].

Memory immune cells are necessary to maintain immunity to microbial pathogens. The presence of Plasmodium-specific memory $\mathrm{T}$ and $\mathrm{B}$ cells in people living in malaria-endemic areas has been reported [3,4]. In a mouse model, memory CD4(+) T cells from chronically infected mice were shown to effectively delay and reduce parasitaemia and pathology [5]. In addition, memory CD8(+) T cells specific for Plasmodium liver-stage antigens prolong protection against malaria [4]. Coincidentally, both antibody and memory $\mathrm{B}$ cell responses to malarial antigens 
in people residing in regions with high Plasmodium transmission rates are stably maintained over time in the absence of re-infection [3,6,7].

CD11c $(+)$ DCs are a major population of antigenpresenting cells that mediate interactions between the innate and adaptive immune responses and play an important role at the host-pathogen interface, including in responses to Plasmodium parasites [8-11]. Pattern recognition receptors expressed by DCs, such as toll-like receptors (TLRs), nod-like receptors, and C-type lectins, recognize distinct conserved microbial molecules [12]. DC maturation is critical in immunity to pathogenic micro-organisms because of the potential of these cells to stimulate differentiation of naïve CD4(+) T cells into various $\mathrm{T}$ helper (Th) cell types, including Th1, Th2, Th17, follicular Th cells, and induced regulatory $\mathrm{T}$ cells $[13,14]$. However, DC function can be compromised during the blood stage of malaria infection, as evidenced by the observation that Plasmodium falciparum-infected red blood cells adhere to human DCs via interaction between $P$. falciparum erythrocyte membrane protein 1 (PfEmP1) and CD36, thus inhibiting DC maturation and subsequently reducing the capacity of DCs to stimulate $\mathrm{T}$ cells $[15,16]$. However, the anti-malarial function of DCs in repeatedly malaria-infected hosts and the longlasting protection of these cells remain unknown. This is the first study to show that CD11c(+) DCs from mice semi-immune to malaria contribute to the prolonged suppression of Plasmodium growth in the blood stage and prevent neurological pathology.

\section{Methods}

\section{Animals and infection}

C57BL/6 J (B6) mice aged six to eight weeks were supplied by SLC Laboratories, Fukuoka, Japan. Semi-immune mice were generated as described elsewhere $[17,18]$. Briefly, C57BL/6 mice were infected with $10^{4} \mathrm{PbA}-\mathrm{pRBCs}$ and then treated on day 5 after infection with intra-peritoneal injection of chloroquine $(20 \mathrm{mg} / \mathrm{kg} /$ day $)$ and pyrimethamine $(20 \mathrm{mg} / \mathrm{kg} /$ day $)$ daily for 7 days. Before subsequent rounds of infection, the parasite-clearance was confirmed and mice were rested for two weeks and then rechallenged with $10^{4} \mathrm{PbA}-\mathrm{pRBCs}$. Mice that underwent three cycles of drug-cured infection became semi-immune. Challenge infections were performed by intraperitoneal injection of $10^{5}$ Plasmodium berghei (ANKA strain) infected RBCs (iRBCs). Plasmodium berghei was selected for its capacity to induce experimental cerebral malaria (ECM) in B6 mice, with neurological signs (ataxia, paralysis, deviation of the head, and convulsions) appearing six to ten days after infection [19]. Three to four months after the third cured infection, semi-immune mice and age-matched controls were finally challenged with $10^{5} \mathrm{P}$. berghei-iRBCs without subsequent treatment.
Approval from the local ethics committee for animal care and research was obtained, and all laboratory and animal practice guidelines of the Animal Centre of the Institute of Tropical Medicine (NEKKEN), Nagasaki, Japan, were adhered to. All experiments were conducted in accordance with local Animal Ethics Committee regulations (Ethical Review Committee at Institute of Tropical Medicine Nagasaki University).

\section{Flow cytometry}

Spleen cells were collected and stained for cell surface antigens. Splenocytes were incubated on ice for $30 \mathrm{~min}$ with fluorochrome-conjugated mAbs against surface antigens. The mAbs used were anti-CD4 PE-cy7, CD8 FITC, CD19 Percp-cy5.5, CD11c APCs, CD11c APC-cy7, B220 PE-cy7, and isotype control Abs (BD Biosciences). Flow cytometric analysis was conducted using FACS Calibur, FACSCanto II, or FACSVerse systems (BD Biosciences), and the resulting data were analysed using Flow Jo software (Tree Star, Inc).

\section{Adoptive transfer}

Three to four months after the third cured infection, non-infected, semi-immune and age-matched naïve B6 mice were sacrificed. Spleens were harvested and homogenized in collagenase $\mathrm{D}$, and the resulting homogenates were resuspended in PBS, pH 7.2. After incubation with $25 \mu \mathrm{l}$ of anti-CD11c microbeads (Miltenyi Biotec), $225 \mu \mathrm{l}$ of cell suspension from one spleen was first sorted for positive selection. The purity of the CD11c(+) fraction was $>95 \%$. A total of $10^{6} \mathrm{CD} 11 \mathrm{c}(+)$ cells from either of semi-immune or naïve mice were intravenously adoptively transferred into six- to eight-weeks-old naïve B6 mice 24 hours before malaria infection with $10^{5}$ P. berghei-iRBCs. Naïve control mice were injected with $200 \mu \mathrm{L}$ of phosphate buffer saline (PBS). Parasitaemia was monitored daily by Giemsa-staining of thin blood smears from day 1 to day 8 post-infection and then every three to four days, and the degree of parasitaemia was expressed as a percentage determined from examination of more than 1,000 RBCs. Mice in each group were monitored daily for symptoms of ECM, such as coma, convulsions, lethargy, tremors, ataxia, hemiplegia, and paraplegia.

\section{ELISA}

Plasma harvesting and measurement of plasma P. bergheispecific IgG subtypes were conducted as described in a previous study [17]. The entire set of experiments was performed at least twice.

\section{Results}

This is the first study demonstrating that splenic CD11c(+) cells from semi-immune mice are capable of prolonged 
inhibition of malaria parasite development and prevention of ECM. This semi-immune mouse model was developed by repeated infection and radical anti-malaria treatment, mimicking the natural boost infection and recovery in humans in areas of intense $P$. falciparum transmission. In previous work, it was shown that mice that undergo three cycles of infection and cure retain resistance to ECM 12 months after the third exposure to malaria.

\section{A history of malaria exposure increased the population of $\mathrm{CD} 4(+), \mathrm{CD} 11 \mathrm{c}(+), \mathrm{B} 220(+) \mathrm{CD} 11 \mathrm{c}(+)$ low cells and the size of $\mathrm{CD} 11 \mathrm{c}(+)$ hi cells in semi-immune mice on day 7 post-infection}

It was found that the percentage of splenic $\mathrm{CD} 4(+)$ and $\mathrm{CD} 11 \mathrm{c}(+)$ cells in semi-immune mice on day 7 postinfection was significantly higher than in infected naïve counterparts (Figure 1A), suggesting a potentially protective cooperation of $\mathrm{CD} 4(+)$ and $\mathrm{CD} 11 \mathrm{c}(+)$ cells against Plasmodium infection. Meanwhile, no significant difference in proportion of $\mathrm{CD} 8(+)$ cells was observed between these two groups of mice (Figure 1A). Thus, this study was carried out with the hypothesis that $\mathrm{CD} 11 \mathrm{c}(+)$ cells from semi-immune mice contributed to immunity against ECM. In murine malaria infection, splenic CD11c(+) cells are considered as splenic CD11c(+) DCs [20]. The role of DCs in prolonged suppression against malaria is still unclear. Hence, the anti-malaria capacity of CD11c(+) DCs and their subtypes was investigated from semi-immune mice in long term after the last immunization. B220(+) CD11c $(+)$ low cells, named as plasmacytoid DCs $[21,22]$, play a critical role in immunity to malaria [21] and in improving the function of conventional DCs [23]. The distribution of this DC subtype in the spleen of semi-immune mice was, therefore, examined. Three months after the third cured malaria infection, B220(+)CD11c(+)low cells in CD19(-) gate accounted for a significantly higher percentage and showed a greater number of cells on day 7 post-infection in infected semi-immune mice than in naïve littermates (2.6 and 1.1\%; 491,031 and 149,699, respectively; $P<0.05)$ (Figure 1B). Meanwhile, no differences in the proportion or number of $\mathrm{B} 220(-) \mathrm{CD} 11 \mathrm{c}(+)$ hi cells (conventional DCs) was observed between these two groups (1.3 and 1.4\%; 222,096 and 127,144, respectively) (Figure 1B). The increase in the proportion of plasmacytoid DCs in semi-immune mice probably enhanced the activation of conventional DCs and, therefore, stimulated the proliferation of $\mathrm{T}$ cells, leading to parasite clearance.

The precise role of plasmacytoid DCs remains unclear. There is, however, growing evidence that the role of plasmacytoid DCs is to support and improve the function of conventional DCs [23]. In non-lethal Plasmodium chabaudi infections, the numbers of both splenic plasmacytoid and conventional DCs are increased, but only conventional DCs upregulate the expression of co-stimulatory molecules and present Plasmodium antigens, resulting in activation of CD4(+) T cells [24,25]. A recent mouse model study showed that up to $70 \%$ of plasmacytoid DCs in the spleen had been in contact with Plasmodium spp. and that a small percentage of these cells were reservoirs of infectious parasites [20]. Significantly, interaction between CD40 on conventional DCs and the CD40 ligand on plasmacytoid DCs leads to IL-12 production by conventional DCs, inducing immune activation [26]. The function of plasmacytoid DCs in malaria should be confirmed in a future study.

In addition, it was found that $\mathrm{CD} 11 \mathrm{c}(+)$ hi cells (conventional DCs) were larger in size on day 7 post-infection in semi-immune mice than naïve counterparts, with forward scatter relative median sizes of 517 and 456, respectively $(P<0.05)$ (Figure $1 C)$. There was no difference in the size of CD11c(+)hi cells on day 0 in these two groups of mice (Figure 1C). The increased size of conventional CD11c $(+)$ hi DCs in the semi-immune mice probably played a role in the beneficial effects of these DCs in the host's immune response to the parasites, as increases in the size of immune cells during malaria infection have been reported. For example, the size and granularity of splenic DCs were shown to increase in mice with malaria [27]. Immature DCs are much smaller in size than mature DCs [28]; therefore, the increased size of $\mathrm{CD} 11 \mathrm{c}(+) \mathrm{hi}$ conventional DCs observed in this study could have functional relevance for immunity against malaria.

\section{Naïve B6 mice receiving splenic CD11c(+) cells from donor semi-immune mice exhibited lower parasitaemia and were partially protected from development of ECM during the acute phase of malaria infection}

To examine the long-lasting anti-malaria role of splenic CD11c(+) DCs derived from semi-immune mice, CD11c(+) cells was isolated with the purity $>95 \%$ (Figure 2A) from the spleens of semi-immune mice three to four months after the third cured infection, as well as from age-matched naïve controls and passively transferred these cells to eightweeks-old naïve mice. As shown in Figure 2B, $61 \%$ of mice receiving $\mathrm{CD} 11 \mathrm{c}(+)$ cells from semi-immune mice (SI11c) were resistant to ECM and survived for 14 days postinfection. Finally, all of these mice succumbed to severe anemia and high parasitaemia around days 28-30 post-infection. Conversely, the survival rates of PBStreated control mice and mice that received $\mathrm{CD} 11 \mathrm{c}(+)$ cells from age-matched naïve mice (Na11c) were significantly lower during the first two weeks of infection, at 28 and $31 \%$, respectively $(P<0.05)$. Splenic CD11c $(+)$ DCs from semi-immune mice with last immunization three months previously partially protected naïve B6 mice against ECM in the passive transfer experiments, confirming the beneficial effects of CD11c(+) DCs in prolonged protection against pathology. $\mathrm{CD} 11 \mathrm{c}(+)$ cells from 

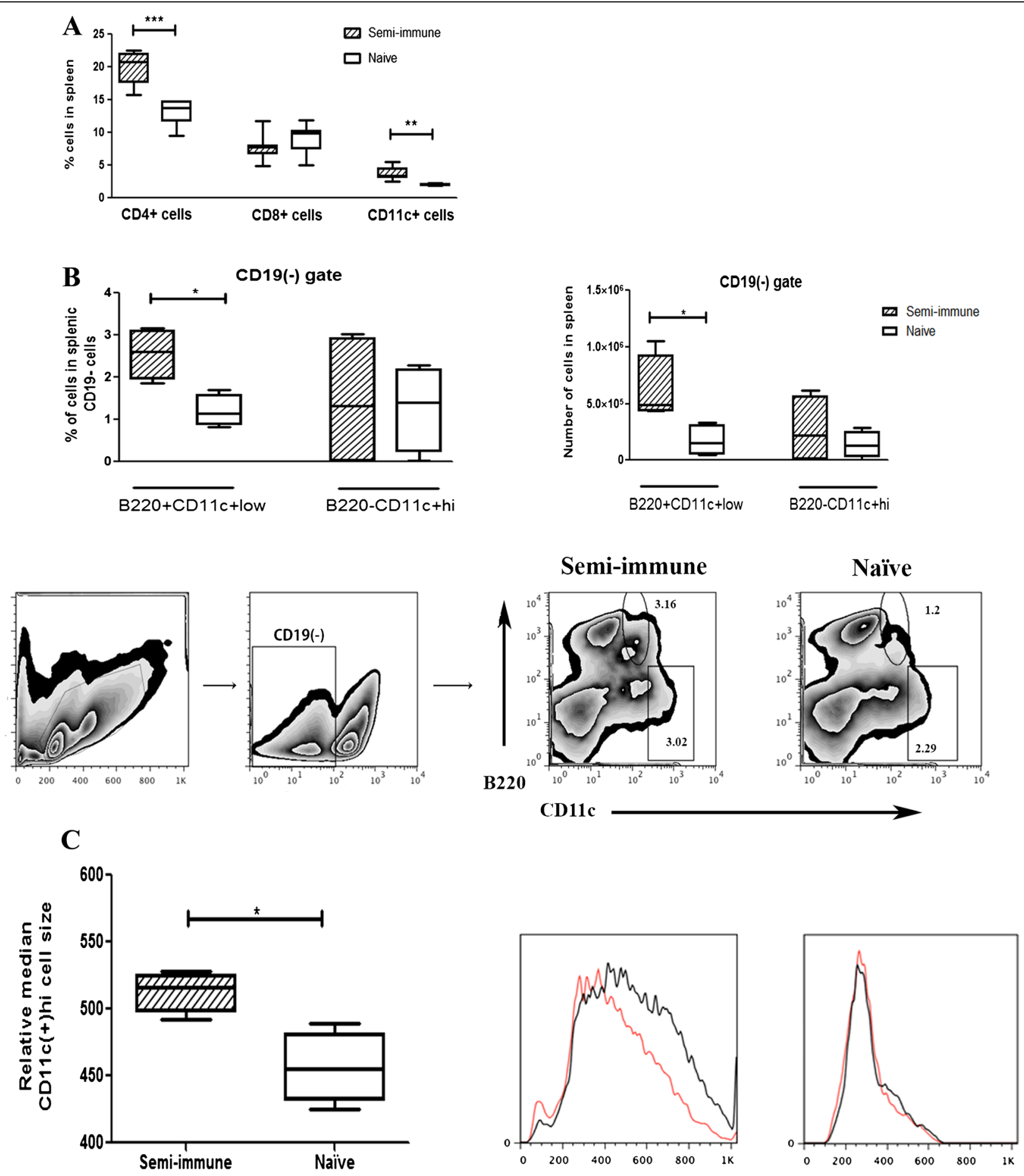

Figure 1 The proportion of $\mathrm{B} 220(+) \mathrm{CD} 11 \mathrm{c}(+)$ low cells was higher and the size of $\mathrm{CD} 11 \mathrm{c}(+)$ hi cells was enhanced on day 7 post-infection in semi-immune mice challenged three months after the third exposure to malaria. (A) Median percentages of splenic CD4(+), CD8(+) and $\mathrm{CD} 11 \mathrm{C}(+)$ cells on day 7 post-infection ( \pm interquartile range) ( $n=6-7$ mice/group). (B) Median percentages and number of splenic B220(+)CD11C $(+)$ low cells and B220(-)CD11C(+)hi cells on day 7 post-infection ( \pm interquartile range) $(n=4$ mice/group). Plots show the gating strategy and data for representatives of these cells on day 7 post-infection in semi-immune and naïve mice. (C) Relative median size of splenic CD11C(+)hi cells on day 7 post-infection ( \pm interquartile range) ( $n=4$ mice/group). Representative histograms regarding the size of CD11c(+)hi cells in semi-immune (black) and naïve (red) mice on day 0 and day 7 post-infection are shown. Data are pooled from two independent experiments (A, B) or representative of two independent experiments (C). ${ }^{*} P<0.05$, Mann-Whitney $U$ test. 

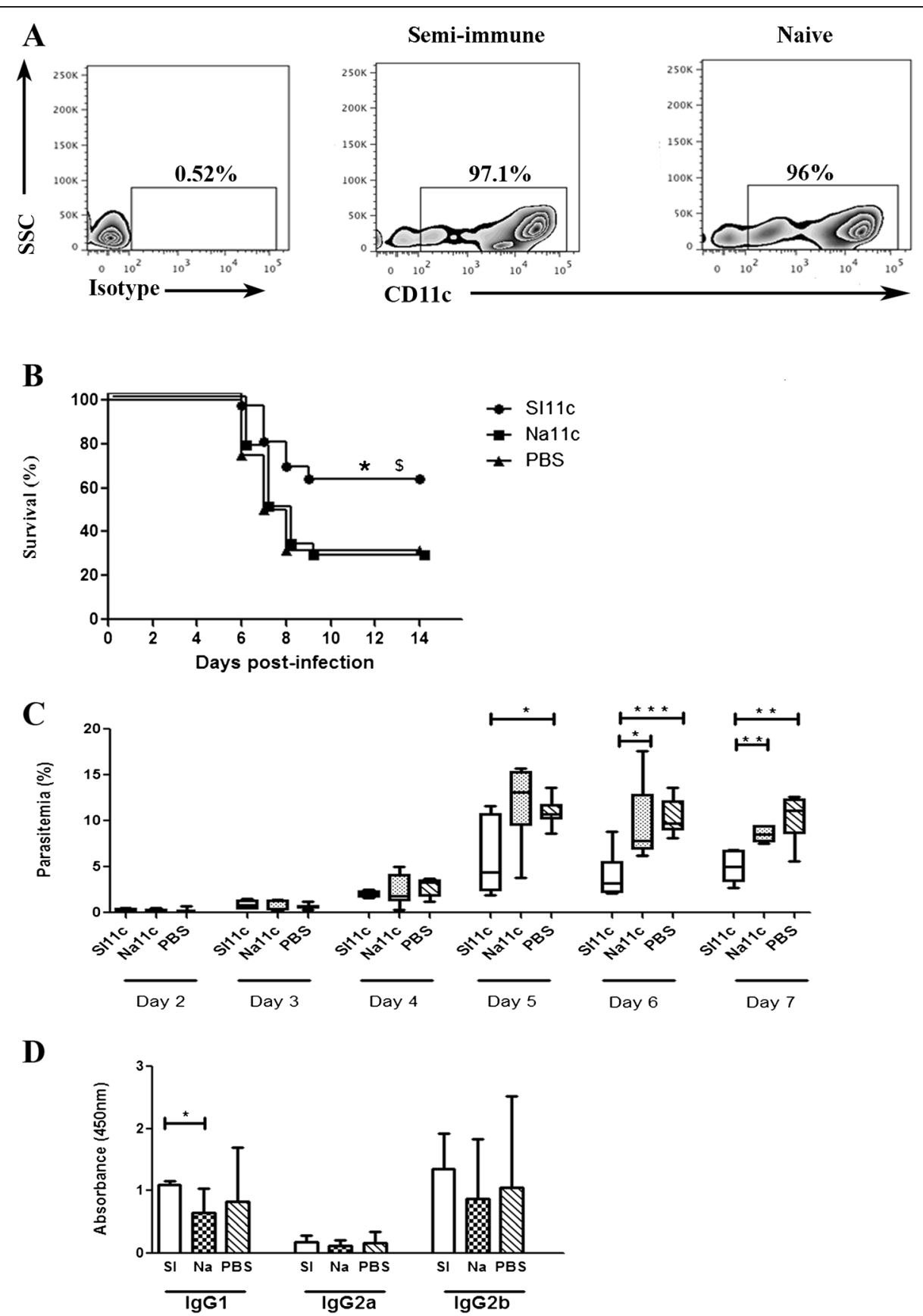

Figure 2 Splenic $\mathrm{CD} 11 \mathrm{c}(+)$ cells from semi-immune mice lowered parasitaemia, enhanced anti-P. berghei lgG1 level and improved the ECM survival rate in recipient mice. (A) Plots show the representative purity of CD11C(+) cells. (B) Survival curves for mice that received $10^{6}$ splenic CD11 $\mathrm{C}(+)$ cells and for PBS-treated control mice $(\mathrm{n}=16-18$ mice/group). Data shown are pooled from three independent experiments. Significant differences between groups as determined by log-rank test are indicated by symbols: ${ }^{*} P<0.05$ for SI11C vs Na11C; $\$ P<0.05$ for SI11C vs PBS. (C) Bar chart showing the median parasitaemia (\%) of recipient mice over the time of infection ( $n=6-9$ mice/group). Parasitaemia (\%) of these groups of mice was performed on days post challenge as indicated and was shown by the box-plots. Data shown are representative of three independent experiments. ${ }^{*} P<0.05 ;{ }^{*} P<0.01 ;{ }^{* *} P<0.001$, Mann-Whitney $U$ test. (D) Median levels of plasma anti-P. berghei lgG1, lgG2a and $\lg \mathrm{G} 2 \mathrm{~b}$ were determined on day 5 post-infection in mice with adoptively transferred DCs. Data are representative of two separate experiments $(n=4$ mice/group). ${ }^{*} P<0.05$, Mann-Whitney $U$ test.

semi-immune mice had a noticeable attenuating effect on parasitaemia in recipients beginning on day 5 post-infection compared with PBS-treated control mice and those that received $\mathrm{CD} 11 \mathrm{c}(+)$ cells from naïve mice (Figure $2 \mathrm{C}$ ). It is known that in malaria infection, DCs containing RBCs expressed higher type I interferon[IFN] levels than those 
without RBCs [21]. Donor CD11c(+) DCs from semiimmune mice, including RBC containing DCs, probably extendedly produced more type I IFN than naïve DCs and then suppressed the parasite proliferation. Consequently, DCs could be one of effective cells for low parasitaemia which was previously observed in semi-immune mice [17]. Prevention of ECM through a lowering of parasitaemia correlated with reduced levels of pathogenic cytokines, chemokines, CD8(+) $\mathrm{T}$ cells, and parasites in the brain has been reported [29-31]. Hence, in the present study, the decrease in parasitaemia mediated by semi-immune DCs probably contributed to the improved survival rate of recipient mice during the acute phase of malaria. CD11c(+) DCs, major innate immune cells, are important in the development of immunity to malaria. Previous studies in mice have demonstrated that in malarial infections that prove lethal, the function of DCs is compromised, but functional DCs improve the survival rate in non-lethal malaria [23,32-35].

\section{Donor CD11c(+) cells from semi-immune mice induced anti-P. berghei lgG1 antibody production in recipient mice during the acute stage of infection}

To identify the type of immune response provoked in mice receiving transferred cells, the levels of plasma IgG subclasses specific to malaria were measured. The level of helper type $2 \mathrm{~T}$ cell (Th2)-mediated anti-P. berghei IgG1 production [36] in semi-immune $\mathrm{CD} 11 \mathrm{c}(+)$ celltreated mice was significantly enhanced compared with naïve $\mathrm{CD} 11 \mathrm{c}(+)$ cell-treated mice $(P<0.05)$. Similar production of helper type $1 \mathrm{~T}$ cell (Th1)-mediated Plasmodium-specific IgG2a and IgG2b [36] was observed in all three mouse groups (Figure 2D). Although the difference was not significant, the $P$. berghei-specific IgG1 level was higher in mice that received semi-immune $\mathrm{CD} 11 \mathrm{c}(+)$ cells than in PBS-treated controls (Figure 2D). These data indicate that Th2 activation via IL-4, Il-5 or IL-13 could be associated with downstream signalling by protective semi-immune DCs. The signalling pathway of semi-immune DCs should be studied further in the future.

\section{Conclusion}

Protective $\mathrm{CD} 11 \mathrm{c}(+)$ DCs from malaria-exposed mice survive for extended periods, despite the absence of further exposure to malaria. These cells prevent development of ECM and lower the degree of parasitaemia. Full elucidation of the role of Plasmodium-exposed CD11c(+) DCs and plasmacytoid DCs in malaria and other infectious diseases requires further study. The findings of the present study may provide the background necessary to prolong the protective immunogenicity of potential vaccine candidates.

\section{Competing interests}

The authors declare that they have no competing interests.

\section{Authors' contributions}

$\mathrm{KH}$ and LQB designed the study, coordinated the experiments, and wrote the manuscript; LQB, DMN and MK performed the experiments and analysed the data. All authors commented on and contributed to writing the manuscript. All authors read and approved the final manuscript.

\section{Acknowledgements}

This work was supported by the Global Centre of Excellence (GCOE), Nagasaki, Japan (to KH (2008-2012)) and Health and Labour Science Research Grants (H25-Kokui-Shitei-001).

\section{Author details}

${ }^{1}$ Department of Immunogenetics, Institute of Tropical Medicine, Nagasaki University, Nagasaki, Japan. ²Department of Clinical Product Development, Institute of Tropical Medicine, Nagasaki University, Nagasaki, Japan. ${ }^{3}$ Centre for International Collaborative Research, Nagasaki University, Nagasaki, Japan. ${ }^{4}$ Animal Research Centre for Tropical Infections, Institute of Tropical Medicine, Nagasaki University, Nagasaki, Japan. ${ }^{5}$ Department of Parasitology, Institute of Tropical Medicine, Nagasaki University, Nagasaki, Japan.

Received: 20 October 2014 Accepted: 26 December 2014 Published online: 28 January 2015

\section{References}

1. Riley EM, Stewart VA. Immune mechanisms in malaria: new insights in vaccine development. Nat Med. 2013;19:168-78.

2. CDC Centers for Disease Control and Prevention. Human Factors and Malaria. 2012.

3. Wipasa J, Suphavilai C, Okell LC, Cook J, Corran PH, Thaikla K, et al. Long-lived antibody and B cell memory responses to the human malaria parasites. Plasmodium falciparum and Plasmodium vivax. PLoS Pathog. 2010;6: e1000770.

4. Krzych U, Dalai S, Zarling S, Pichugin A. Memory CD8T cells specific for Plasmodia liver-stage antigens maintain protracted protection against malaria. Front Immunol. 2012;3:370.

5. Stephens R, Langhorne J. Effector memory Th1 CD4 T Cells are maintained in a mouse model of chronic malaria. PLoS Pathog. 2010;6:e1001208.

6. Ndungu FM, Olotu A, Mwacharo J, Nyonda M, Apfeld J, Mramba LK, et al. Memory B cells are a more reliable archive for historical antimalarial responses than plasma antibodies in no-longer exposed children. Proc Natl Acad Sci U S A. 2012;109:8247-52.

7. Stevenson MM, Ing R, Berretta F, Miu J. Regulating the adaptive immune response to blood-stage malaria: role of dendritic cells and $\mathrm{CD} 4^{+}$Foxp $3^{+}$ regulatory T cells. Int J Biol Sci. 2011;7:311-22.

8. Banchereau J, Briere F, Caux C, Davoust J, Lebecque S, Liu YJ, et al. Immunobiology of den-dritic cells. Annu Rev Immunol. 2000;18:767-811.

9. Mellman I, Steinman RM. Dendritic cells: specialized and regulated antigen processing machines. Cell. 2001;106:255-8.

10. Rescigno M, Borrow P. The host-pathogen interaction: new themes from dendritic cell biology. Cell. 2001;106:267-70.

11. Urban BC, Ing R, Stevenson MM. Early interactions be-tween blood-stage Plasmodium parasites and the immune sys-tem. Curr Top Microbiol Immunol. 2005;297:25-70.

12. Iwasaki A, Medzhitov R. Regulation of adaptive immunity by the innate immune system. Science. 2010;327:291-5.

13. Zhu J, Paul WE. CD4 T cells: fates, functions, and faults. Blood. 2008;112:1557-69.

14. Coquerelle $C$, Moser M. DC subsets in positive and negative regulation of immunity. Immunol Rev. 2010;234:317-34.

15. Urban BC, Ferguson DJ, Pain A, Willcox N, Plebanski M, Austyn JM, et al. Plasmodium falciparum-infected erythrocytes modulate the maturation of dendritic cells. Nature. 1999:400:73-7.

16. Urban BC, Willcox N, Roberts DJ. A role for CD36 in the regulation of dendritic cell function. Proc Natl Acad Sci U S A. 2001;98:8750-5.

17. Bao LQ, Huy NT, Kikuchi M, Yanagi T, Senba M, Shuaibu MN, et al. CD19(+) $B$ cells confer protection against experimental cerebral malaria in semi-immune rodent model. PLoS One. 2013;8:e64836. 
18. Evans KJ, Hansen DS, van Rooijen N, Buckingham LA, Schofield L. Severe malarial anemia of low parasite burden in rodent models results from accelerated clearance of uninfected erythrocytes. Blood. 2006;107:1192-9.

19. Amani V, Boubou Ml, Pied S, Marussig M, Walliker D, Mazier D, et al. Cloned lines of Plasmodium berghei ANKA differ in their abilities to induce experimental cerebral malaria. Infect Immun. 1998;66:4093-9.

20. Wykes MN, Kay JG, Manderson A, Liu XQ, Brown DL, Richard DJ, et al. Rodent blood-stage Plasmodium survive in dendritic cells that infect naive mice. Proc Natl Acad Sci U S A. 2011;108:11205-10.

21. Wu J, Tian L, Yu X, Pattaradilokrat S, Li J, Wang M, et al. Strain-specific innate immune signaling pathways determine malaria parasitemia dynamics and host mortality. Proc Natl Acad Sci U S A. 2014;111:E511-520.

22. Smit JJ, Rudd BD, Lukacs NW. Plasmacytoid dendritic cells inhibit pulmonary immunopathology and promote clearance of respiratory syncytial virus. J Exp Med. 2006:203:1153-9.

23. Wykes MN. Are plasmacytoidendritic cells the misguided sentinels of malarial immunity? Trends Parasitol. 2012;28:182-6.

24. Sponaas AM, Cadman ET, Voisine C, Harrison V, Boonstra A, O'Garra A, et al. Malaria infection changes the ability of splenic dendritic cell populations to stimulate antigen-specifi c T cells. J Exp Med. 2006;203:1427-33.

25. Voisine C, Mastelic B, Sponaas AM, Langhorne J. Classical CD11C + dendritic cells, not plasmacytoid dendritic cells, induce T cell responses to Plasmodium chabaudi malaria. Int J Parasitol. 2010;40:711-9.

26. Kuwajima S, Sato T, Ishida K, Tada H, Tezuka H, Ohteki T. Interleukin 15-dependent crosstalk between conventional and plasmacytoid dendritic cells is essential for CpG-induced immune activation. Nat Immunol. 2006;7:740-6.

27. Millington OR, Di Lorenzo C, Phillips RS, Garside P, Brewer JM. Suppression of adaptive immunity to heterologous antigens during Plasmodium infection through hemozoin-induced failure of dendritic cell function. J Biol. 2006;5:5.

28. Dumortier H, van Mierlo GJ, Egan D, van Ewijk W, Toes RE, Offringa R, et al. Antigen presentation by an immature myeloid dendritic cell line does not cause CTL deletion in vivo, but generates CD8+ central memory-like T cells that can be rescued for full effector function. J Immunol. 2005;175:855-63.

29. Haque A, Best SE, Unosson K, Amante FH, de Labastida F, Anstey NM, et al. Granzyme B expression by CD8+ T cells is required for the development of experimental cerebral malaria. J Immunol. 2011;186:6148-56.

30. Baptista FG, Pamplona A, Pena AC, Mota MM, Pied S, Vigário AM. Accumulation of Plasmodium berghei-infected red blood cells in the brain is crucial for the development of cerebral malaria in mice. Infect Immun. 2010;78:4033-9.

31. McQuillan JA, Mitchell AJ, Ho YF, Combes V, Ball HJ, Golenser J, et al. Coincident parasite and CD8 T cell sequestration is required for development of experimental cerebral malaria. Int J Parasitol. 2011;41:155-63.

32. Wykes MN, Good MF. What really happens to dendritic cells during malaria? Nat Rev Microbiol. 2008;6:864-70.

33. Wykes M, Keighley C, Pinzon-Charry A, Good MF. Dendritic cell biology during malaria. Cell Microbiol. 2007;9:300-5.

34. Stevenson MM, Ing R, Berretta F, Miu J. Regulating the adaptive immune response to blood-stage malaria: role of dendritic cells and CD4Foxp3 regulatory T cells. Int J Biol Sci. 2011;7:1311-22.

35. Lundie RJ. Antigen presentation in immunity to murine malaria. Curr Opin Immunol. 2011;23:119-23.

36. Kumar S, Jones TR, Oakley MS, Zheng H, Kuppusamy SP, Taye A, et al. CpG oligodeoxynucleotide and montanide ISA 51 adjuvant combination enhanced the protective efficacy of a subunit malaria vaccine. Infect Immun. 2004;72:949-57.

\section{Submit your next manuscript to BioMed Central and take full advantage of:}

- Convenient online submission

- Thorough peer review

- No space constraints or color figure charges

- Immediate publication on acceptance

- Inclusion in PubMed, CAS, Scopus and Google Scholar

- Research which is freely available for redistribution

Submit your manuscript at www.biomedcentral.com/submit 